\title{
Repeated Ethanol Intoxication Induces Behavioral Sensitization in the Absence of a Sensitized Accumbens Dopamine Response in C57BL/6J and DBA/2J Mice
}

\author{
Agustin Zapata*, 1,2, Rueben A Gonzales ${ }^{2}$ and Toni S Shippenberg' \\ 'Integrative Neuroscience Section, Behavioral Neuroscience Branch National Institute on Drug Abuse Intramural Research Program, Baltimore, \\ MD, USA; ${ }^{2}$ College of Pharmacy, University of Texas at Austin, Austin, TX, USA
}

\begin{abstract}
Repeated exposure to drugs of abuse results in an increased sensitivity to their behavioral effects, a phenomena referred to as behavioral sensitization. It has been suggested that the same neuroadaptations underlying behavioral sensitization contribute to the maintenance and reinstatement of addiction. Dysregulation of dopamine (DA) neurotransmission in the mesoaccumbens system is one neuroadaptation that is thought to lead to the compulsive drug-seeking that characterizes addiction. Evidence that sensitization to psychostimulants and opiates is associated with an enhancement of drug-evoked DA levels in the nucleus accumbens has also been obtained. Like other drugs of abuse, the acute administration of ethanol $(\mathrm{ETOH})$ stimulates DA release in this brain region. Moreover, repeated ETOH experience results in an enhanced behavioral response to a subsequent ethanol challenge. Data regarding the influence of repeated ethanol intoxication and withdrawal upon mesoaccumbal DA neurotransmission is limited. Studies examining ETOH-evoked alterations in mesoaccumbal DA neurotransmission as a function of withdrawal duration are lacking. The present experiments quantified basal and ethanol-evoked DA levels 14 days and $24 \mathrm{~h}$ following the cessation of a repeated ETOH intoxication protocol, which results in sensitization to the locomotor activating effects of ethanol. Locomotor activity was assessed in parallel groups of animals. Studies were conducted in two mouse strains, C57BL/6) and DBA/2], which differ in their behavioral responses to ETOH. The results indicate the development of transient tolerance to both $\mathrm{ETOH}$-induced behavioral activation and evoked accumbens DA release at early withdrawal. Moreover, no enhanced DA response to a subsequent ETOH challenge could be demonstrated in ETOH experienced animals 2 weeks after withdrawal, in spite of the observation of clear behavioral sensitization at this time point. These results suggest that, at least in the case of ethanol, sensitization of the DA mesolimbic system may not be necessary for the development of behavioral sensitization. Neuropsychopharmacology (2006) 3 I, 396-405. doi:I 0. I038/sj.npp. I 300833; published online 20 July 2005
\end{abstract}

Keywords: inbred strains; microdialysis; ventral striatum; chronic ethanol; addiction; alcohol

\section{INTRODUCTION}

Repeated exposure to various drugs of abuse results in an enhancement of their behavioral effects. This phenomenon, referred to as behavioral sensitization, is manifested in rodents as an increase in drug-evoked locomotor activity after repeated drug administration. Behavioral sensitization is proposed to result from some of the same neuroadaptations that are triggered by repeated drug exposure and that lead to the development of compulsive drug use

*Correspondence: Dr A Zapata, Integrative Neuroscience Section, Behavioral Neuroscience Branch, NIDA IRP, 5500 Nathan Shock Drive, Baltimore, MD 21224, USA, Tel: + I 410550 2475, Fax: + I 410550 |692, E-mail: Azapata@intra.nida.nih.gov

Received 21 February 2005; revised 22 May 2005; accepted 31 May 2005

Online publication: 20 June 2005 at http://www.acnp.org/citations/ Npp062005050 I I 6/default.pdf
(Kalivas et al, 1998). One neuroadaptation that has received considerable attention involves the effects of repeated drug experience on the mesoaccumbal dopaminergic system. Dopamine (DA) is thought to be important for both the induction and the expression of behavioral sensitization (Robinson and Berridge, 1993; Pierce and Kalivas, 1997). Drugs of abuse stimulate the activity of mesoaccumbal DA neurons and this effect increases upon repeated drug exposure. Sensitization of the mesoaccumbal DA system after repeated drug administration has been demonstrated for most drugs of abuse, including psychostimulants (Kalivas and Duffy, 1993a; Cadoni et al, 2000b; Zapata et al, 2003), opiates (Cadoni and Di Chiara, 1999), and nicotine (Reid et al, 1996; Cadoni and Di Chiara, 2000a). This enhanced accumbens DA response after repeated drug experience has been proposed to be one neuroadaptation important for the maintenance and reinstatement of compulsive drug-seeking behavior (Robinson and Berridge, 1993). 
Similar to other drugs of abuse, acute ethanol (ETOH) administration stimulates DA release in the nucleus accumbens (Imperato and Di Chiara, 1986; Yoshimoto et al, 1992; Yim et al, 1998). DA receptor antagonists attenuate the locomotor-activating effects of acute ETOH, suggesting an important role of mesoaccumbal DA neurons in mediating the acute effects of ETOH (Liljequist et al, 1981; Risinger et al, 1992; Shen et al, 1995). Behavioral sensitization after repeated ETOH administration has been demonstrated in rodents (Phillips et al, 1994; Hoshaw and Lewis, 2001; Fish et al, 2002). The role of DA neurons, however, in mediating the development of this adaptive response has received very little attention. Although behavioral sensitization to ETOH is associated with an enhancement of electrically evoked DA release in nucleus accumbens slices (Nestby et al, 1997, 1999), systemic administration of the DA receptor antagonist haloperidol failed to modify the sensitization that develops to the locomotor-activating effects of ETOH (Broadbent et al, 1995).

The contribution of mesoaccumbal DA neurons to the development and/or expression of ETOH dependence is also unclear. Several reports have shown a pronounced decrease in basal extracellular DA levels in the nucleus accumbens during the initial phase of ETOH withdrawal (Diana et al, 1993; Weiss et al, 1996). However, these studies were performed within $6-12 \mathrm{~h}$ after the cessation of ETOH administration. Therefore, it is not known whether these changes in accumbens DA tone are long lived. Such information, however, is important in view of a wealth of data demonstrating that the neurochemical response to other drugs of abuse varies as a function of withdrawal duration. Information regarding the reactivity of the mesoaccumbens DA system to renewed ETOH exposure in individuals with a prior history of repeated ETOH administration is also lacking.

Repeated ETOH intoxication and withdrawal in rodents is thought to model some of the consequences of repeated binge drinking in long-term alcoholics (Becker, 1994; Duka et al, 2004). As such, it may provide an effective animal model for quantifying alterations in behavior and neurochemistry that are associated with chronic ethanol use. Accordingly, the present microdialysis studies were performed in the mouse to characterize the effects of repeated ETOH intoxication and withdrawal on basal and ETOHevoked DA levels in the nucleus accumbens. Since the effects of ETOH are known to be dependent on genotype, studies were performed in two mouse strains known to differ in their responses to ETOH: the C57BL/6J and the $\mathrm{DBA} / 2 \mathrm{~J}$ strains. DBA/2J mice show strong locomotor activation in response to ETOH (Phillips et al, 1994) and readily display ETOH-induced conditioned place preference (Cunningham et al, 1992). They exhibit a robust withdrawal syndrome (measured by handling-induced convulsions (HICs)) (Crabbe et al, 1983; Crabbe and Belknap, 1993) and avoid ETOH consumption in a two bottle choice paradigm (Crabbe et al, 1983). In contrast, C57BL/6J mice readily develop preference for ETOH, but show little or no locomotor activation in response to an acute ETOH challenge, no ethanol-induced conditioned place preference, and a mild withdrawal syndrome (Cunningham et al, 1992; Crabbe and Belknap, 1993; Phillips et al, 1994). To confirm that our repeated ETOH treatment was sufficient to induce behavioral sensitization, we also examined the effects of chronic ETOH intoxication and withdrawal on ETOHevoked locomotor activity. Blood ETOH concentrations were measured in order to evaluate the contribution of possible metabolic adaptations to the results obtained.

\section{MATERIALS AND METHODS}

\section{Animals}

Experimentally naïve, male $\mathrm{C} 57 \mathrm{BL} / 6 \mathrm{~J}$ and $\mathrm{DBA} / 2 \mathrm{~J}$ mice (Jackson laboratory, Bar Harbor, ME) between 6 and 10 weeks old were housed two per cage in a temperaturecontrolled vivarium. All animals were acclimated to their home cages and to the light-dark cycle for at least 7 days prior to testing. Water and food were continuously available for the mice in their home cages. Experiments were conducted during the light phase of a 12-h light/dark cycle. The facilities in which the animals were maintained are fully accredited by the American Association for the Accreditation of Laboratory Animal Care (AAALAC), and the studies described here were conducted in accordance with the Guide for Care and Use of Laboratory Animals of the NIH. All experimental protocols were approved by the NIDA Institutional Animal Care and Use Committee.

\section{Repeated Ethanol Intoxication and Withdrawal}

The repeated ETOH intoxication and withdrawal procedure was adapted from that described previously by Becker et al (Becker, 1994; Becker et al, 1997) with minor modifications. Mice were injected (i.p.) with a loading dose of $1.68 \mathrm{~g} / \mathrm{kg}$ ETOH (11.2\% (w/v), $15 \mathrm{ml} / \mathrm{kg}$ injection volume) and the alcohol dehydrogenase inhibitor, pyrazole $(68 \mathrm{mg} / \mathrm{kg})$, in order to achieve stable blood ETOH concentrations. They were then transferred to their home cages, which were placed inside an ETOH vapor chamber $(60 \times 60 \times 45 \mathrm{~cm})$. The ETOH concentration in the air inside the chamber was $16.2 \pm 0.3 \mathrm{mg} / \mathrm{l}$ (mean \pm SEM, $n=28$ experiments) and was achieved by continuously pumping a mixture of normal air $(51 / \mathrm{min})$ and air saturated in ETOH by bubbling in a 11 bottle containing $750 \mathrm{ml}$ of $95 \%$ ETOH $(0.951 / \mathrm{min})$. Mice remained in the ETOH chambers for $16 \mathrm{~h}$ per day (06:00-10:00) followed by $8 \mathrm{~h}$ of withdrawal. Mice were placed in the chambers on 4 consecutive days and each exposure was preceded by injection of $\mathrm{ETOH}$ and pyrazole Control mice received saline injections and were exposed to normal air in the chambers. Locomotor activity testing and microdialysis experiments were conducted in separate groups of mice 1 and 14 days following the last treatment session. The experimental timeline is summarized in Figure 1.

\section{Handling-Induced Convulsions}

Withdrawal severity was assessed by the HIC scale, $8 \mathrm{~h}$ following the termination of the last ETOH exposure session. The scale used was identical to that described in previous studies (Becker et al, 1997). 


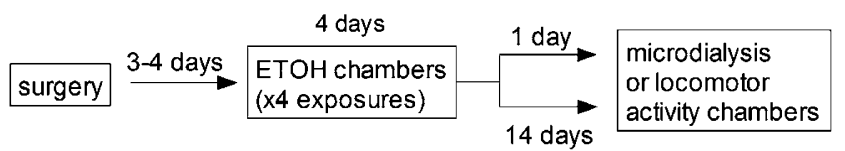

Figure I Diagram showing the experimental timeline used in the present studies. Animals used for microdialysis studies went through surgery before exposure to the ethanol chambers. The animals used for the locomotor activity studies did not receive any surgery. All animals went through the ethanol vapor exposure regimen (or normal air for controls) and then they were divided into two groups. The first group was tested at I day of withdrawal and the second group was tested at 14 days of withdrawal (see details in the Materials and methods section).

\section{Microdialysis}

The microdialysis procedure has been described in detail elsewhere (Zapata et al, 2001). Briefly, mice were anesthetized with sodium pentobarbital $(75 \mathrm{mg} / \mathrm{kg}$ i.p.) and a microdialysis guide cannula (CMA 11, CMA Microdialysis, North Chelmsford, MA) was stereotaxically implanted above the nucleus accumbens (coordinates were: anteroposterior $+1.5 \mathrm{~mm}$, lateral $-1.0 \mathrm{~mm}$, ventral $-3.8 \mathrm{~mm}$ relative to bregma). After surgery, mice were individually housed in the colony room and allowed to recover for 3-4 days before the experiments.

Microdialysis was conducted 1 or 14 days after the last ETOH exposure. Separate animals were used at each abstinence time point. The night before the microdialysis experiment, the microdialysis probe $(1 \mathrm{~mm}$ membrane length, CMA/11, CMA microdialysis, North Chelmsford, MA) was manually inserted into the guide cannula and perfused with artificial cerebrospinal (aCSF: $145 \mathrm{mM} \mathrm{NaCl}$, $2.8 \mathrm{mM} \mathrm{KCl}, 1.2 \mathrm{mM} \mathrm{CaCl}, 1.2 \mathrm{mM} \mathrm{MgCl}_{2}$ and $5.4 \mathrm{mM}$ D-glucose) via gas tight microsyringes and a microsyringe pump. The mice were placed in the microdialysis cages and allowed to habituate overnight. The next morning, fresh aCSF was loaded into the microsyringes and perfused for $1 \mathrm{~h}$ before commencement of the experiment. A flow rate of $0.6 \mu \mathrm{l} / \mathrm{min}$ was used for all the studies. Consecutive $20 \mathrm{~min}$ dialysate samples were collected and stored at $-80^{\circ} \mathrm{C}$ until analysis. After four baseline samples, mice received an acute i.p. injection of ETOH $(2.0 \mathrm{~g} / \mathrm{kg}, 15 \%(\mathrm{w} / \mathrm{v}), 13.3 \mathrm{ml} / \mathrm{kg})$ and six more samples were collected. Dialysate DA content was determined by HPLC coupled to electrochemical detection (Zapata et al, 2001). After the experiment, the animals were killed by pentobarbital overdose and the brains dissected and frozen at $-80^{\circ} \mathrm{C}$. Probe placement was verified histologically using $30 \mu \mathrm{m}$ coronal sections obtained in a cryostat. Only animals with probe placements restricted to the nucleus accumbens were included in the analysis.

Since the control animals were not treated with pyrazole, an additional experiment was performed in order to test whether pyrazole treatment alters ETOH-evoked accumbens DA when compared to repeated injections of saline. The same pyrazole treatment schedule as the one employed in the ETOH exposure protocol was used $(68 \mathrm{mg} / \mathrm{kg}$ i.p. or saline, 1 daily injection $\times 4$ days, microdialysis was performed $40 \mathrm{~h}$ after the last pyrazole injection).

\section{Locomotor Activity}

The effects of ETOH withdrawal on basal and ETOH-evoked locomotor activity were evaluated 1 and 14 days after the last ETOH exposure in parallel groups of mice. Monitoring of locomotor activity was performed in $40 \mathrm{~cm}^{3}$ Digiscan Animal Activity monitor cages (Omnitech Electronics, Columbus, $\mathrm{OH}$ ) equipped with infrared beams aimed at photoelectric detectors placed $2.6 \mathrm{~cm}$ apart along the perimeter. The detectors were capable of sensing movement at a height of up to $20 \mathrm{~mm}$ off the floor. Mice were placed in the activity cages and allowed to habituate for $2 \mathrm{~h}$. They received an i.p. injection of saline $(13.3 \mathrm{ml} / \mathrm{kg})$ and were returned to the activity cages for $1 \mathrm{~h}$. They then received an i.p. injection of ETOH $(2.0 \mathrm{~g} / \mathrm{kg}, 15 \%(\mathrm{w} / \mathrm{v}), 13.3 \mathrm{ml} / \mathrm{kg})$ and were returned to the activity cages for one additional hour. Locomotor activity was recorded at $5 \mathrm{~min}$ intervals during the experiment ( $2 \mathrm{~h}$ habituation, $1 \mathrm{~h}$ saline, $1 \mathrm{~h} \mathrm{ETOH}$ ) using a Versamax Analyser and software (Accuscan Instruments Inc., Columbus, $\mathrm{OH}$ ). The two mouse strains were run in independent experiments. For each withdrawal time point, four ETOH and four control (air-exposed) mice were simultaneously tested.

\section{Blood ETOH Concentrations}

Blood samples $(40 \mu \mathrm{l})$ were obtained from the retro-orbital sinus. Both, control and ETOH-exposed mice were sampled immediately after the last exposure in the chambers. In addition, mice used in the locomotor activity experiments were sampled a second time immediately after the completion of the locomotor activity experiment $(1 \mathrm{~h}$ post-ETOH challenge). Blood samples were precipitated in $3 \%$ perchloric acid (200 $\mu$ l final volume) and stored at $4^{\circ} \mathrm{C}$ until analysis. ETOH was quantified by the alcohol dehydrogenase assay. Samples $(20 \mu \mathrm{l})$ were incubated in duplicate in $1 \mathrm{ml}$ of $0.5 \mathrm{M}$ Tris-Cl buffer ( $\mathrm{pH}$ 8.8) containing $5.5 \mu \mathrm{g} / \mathrm{ml}$ of alcohol dehydrogenase and $1.5 \mathrm{mM} \beta$-nicotinamide adenine dinucleotide ( $\beta$-NAD) for $40 \mathrm{~min}$ at room temperature. Accumulation of $\beta$-NADH was measured by reading sample absorbance at $340 \mathrm{~nm}$. The ETOH concentration in the samples was estimated by using a standard calibration curve.

\section{Data Analysis}

Control animals were run in parallel with ETOH withdrawn animals, and tested either 1 day or 14 days after air exposure. However, since no differences between the control groups in either locomotor activity or dialysis experiments were observed at the two abstinence time points, the data for both control groups were pooled. Thus, the withdrawal condition was assessed as three levels: control (no previous ETOH experience, but submitted to the same manipulations as the experimental animals), early withdrawal (1 day after ETOH withdrawal), and late withdrawal (14 days after ETOH withdrawal).

Dialysate DA concentrations are expressed as a percentage of baseline. Basal DA for each animal was calculated as the average concentration of the four basal samples. For statistical analysis of ETOH-evoked DA levels, an area under the curve (AUC) value for the six samples collected after ETOH administration was calculated for each animal using absolute DA concentrations (nM) according to the standard trapezoid method. The formula used was AUC $=$ $\left(0.5 \times\left(B+S_{1}\right) \times d+0.5 \times\left(S_{1}+S_{2}\right) \times d+0.5 \times\left(S_{2}+S_{3}\right) \times d+\right.$ $\left.\left.\cdots 0.5 \times\left(S_{n-1}+S_{n}\right) \times d\right)-(B \times d \times n)\right)$, where $B$ is the 
average basal concentration (nM), $S_{x}$ are the concentrations (nM) of each fraction collected during drug challenge, $n$ the total number of fractions collected during drug challenge, and $d$ the duration of each fraction (in min). The effect of ETOH withdrawal on basal and ETOH-evoked DA levels was assessed using a two-factor ANOVA with withdrawal condition as one factor and strain as the second factor. Post hoc comparisons among groups were performed by the Student-Newman-Keuls multiple comparison test.

The locomotor activity data are expressed as ambulatory distance traveled $(\mathrm{cm})$ in each $5 \mathrm{~min}$ period. For statistical analysis, saline and ETOH-evoked locomotor activity was calculated as the total distance traveled for $20 \mathrm{~min}$ after the saline or the ETOH challenge. Since the data were not normally distributed, the effect of the withdrawal condition within each mouse strain was analyzed by Kruskal-Wallis analysis of variance on ranks, followed by the Dunn's test for multiple post hoc comparisons. Differences between mouse strains were determined by Mann-Whitney's tests. Although nonparametric statistics were used for data analysis, the data are presented as mean \pm SEM in the figures.

Blood ETOH concentrations in response to chamber exposure were analyzed as a function of strain using the Student's $t$-test. Blood ETOH concentrations in response to the challenge dose of ETOH were analyzed by a two-factor ANOVA (withdrawal condition $\times$ strain). Strain differences in withdrawal scores were evaluated by a Mann-Whitney rank-sum test.

\section{RESULTS}

\section{Blood ETOH Concentrations}

Blood ETOH concentrations $1 \mathrm{~h}$ after the $2.0 \mathrm{~g} / \mathrm{kg}$ ETOH challenge are shown in Figure 2a. Blood ETOH concentrations in response to the challenge dose of ETOH did not differ between strains in control mice. However, in animals with a prior history or ETOH exposure, blood levels differed as a function of strain and withdrawal condition. Two-factor ANOVA indicated a main effect of withdrawal condition $\left(\mathrm{F}_{(2,75)}=7.35, p=0.001\right)$ and a significant strain by withdrawal condition interaction $\left(\mathrm{F}_{(2,75)}=8.18, p<0.001\right)$. However, no significant main effect of strain was seen. Post hoc analysis revealed that blood ETOH levels produced by the challenge injection of ETOH in ETOH-exposed C57BL/ $6 \mathrm{~J}$ mice were lower than same strain controls at the 1 day time point, suggesting a faster metabolism of ETOH during early withdrawal in this strain. This effect was transient since it was not apparent in the 14-day withdrawal group. In contrast, in the DBA/2J mice no difference relative to controls was detected in the 1-day withdrawal group. Interestingly, however, blood ETOH levels were significantly lower at 14 days withdrawal.

Figure $2 \mathrm{~b}$ shows the blood ETOH concentrations obtained immediately after the last exposure to the ETOH vapor chambers. C57BL/6J mice showed significantly lower blood ETOH levels than their DBA/2J counterparts $(\mathrm{t}=-2.80$ with $45 \mathrm{df}, p=0.007)$. This is consistent with the idea that C57BL/6J mice, but not DBA/2J mice, have lower blood ETOH concentrations after chronic ETOH exposure.
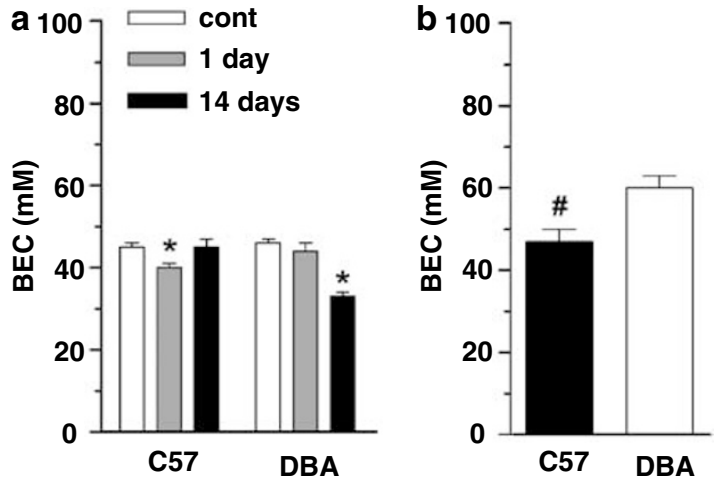

Figure 2 Blood ETOH concentrations (BEC) after the locomotor activity test I h after ETOH $2 \mathrm{~g} / \mathrm{kg}$ i.p. (a) or immediately after the last ETOH vapor exposure (b). Values are mean \pm SEM, $*$ p $<0.05$ vs control, Student-Newman-Keuls. ${ }^{\#} p<0.05$ vs the other genotype, Student's $t$-test. Number of animals per group was: Figure Ia, C57BL/6] $(n=20,13$, and 6) and DBA/2 $(n=20,15$, and 7) for control, I- and 14-day withdrawal groups, respectively. Figure Ib, $n=19$ and 28 for C57BL/6] and DBA/2], respectively.

\section{Withdrawal Severity}

Regardless of strain, none of the control (air-exposed), mice showed any withdrawal signs. Significant strain-related differences in withdrawal severity, as measured by the HIC score, were observed in ETOH-exposed mice. DBA/2J mice showed significantly higher HIC scores $8 \mathrm{~h}$ following the last exposure to the ETOH chambers when compared to C57BL/ $6 \mathrm{~J}$ mice $(3.12 \pm 0.15$ (17) vs $1.26 \pm 0.15$ (19), mean $\pm \operatorname{SEM}(n)$, $\mathrm{t}=469, p<0.001$ Mann-Whitney rank-sum test). The lower score of $\mathrm{C} 57 \mathrm{BL} / 6 \mathrm{~J}$ mice indicates that although the repeated $\mathrm{ETOH}$ treatment produces withdrawal, it is mild relative to that of DBA/2J.

Exposure to the ETOH treatment resulted in significant mortality (nine out of 28 and six out of 38 mice in C57BL/6J and DBA/2J mice, respectively). The mortality rate was not significantly different between the two mouse strains $\left(\chi^{2}=1.612 ; \mathrm{df}=1, p=0.204\right)$. No mortality was observed in control animals (zero out of 20 in both C57BL/6J and $\mathrm{DBA} / 2 \mathrm{~J}$ mice).

\section{Locomotor Activity}

The time course of the locomotor activity experiments is shown in Figure 3. Statistical analysis indicated no differences between strains in the response to a saline injection (Figure 4a, $\mathrm{t}=455, p=0.92$, Mann-Whitney's rank-sum test). There was, however, a main effect of withdrawal condition in both the C57BL/6J $(\mathrm{H}=7.93$, with $2 \mathrm{df}, p=0.019$, Kruskal-Wallis ANOVA on ranks) and the $\mathrm{DBA} / 2 \mathrm{~J}$ mouse strains $(\mathrm{H}=13.09$, with $2 \mathrm{df}, p=0.001$, Kruskal-Wallis ANOVA on ranks). This effect was due to a decreased locomotor response to saline in both strains at the 1-day withdrawal time point.

When the effects of the $2 \mathrm{~g} / \mathrm{kg}$ i.p. ETOH challenge on locomotor activity were analyzed, significantly greater locomotor response was detected in DBA/2J mice compared with $\mathrm{C} 57 \mathrm{BL} / 6 \mathrm{~J}$ mice (Figure $4 \mathrm{~b}, \mathrm{t}=235, p<0.001$, control C57BL/6J vs control DBA/2J, Mann-Whitney's rank-sum 
C57BL/6J
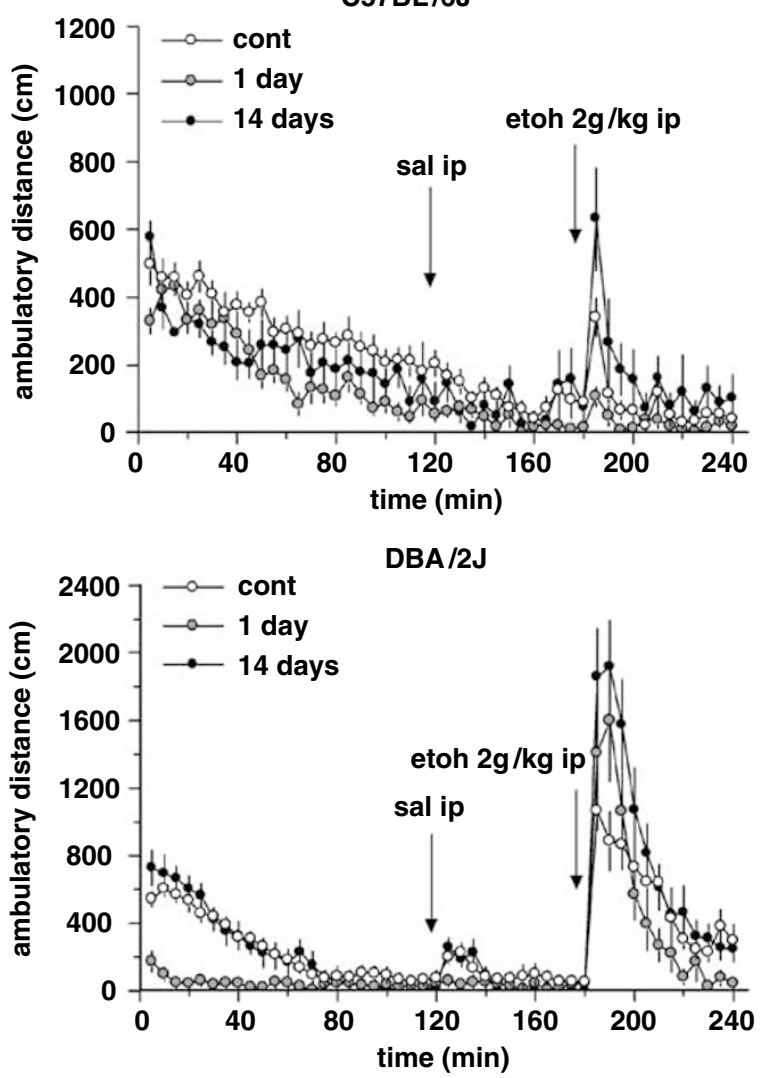

Figure 3 Time course of the effects of a saline and ETOH $2 \mathrm{~g} / \mathrm{kg}$ i.p. challenge on locomotor activity in naive or ETOH withdrawn (I or I 4 day) C57BL/6) or DBA/2 I mice. Values are mean \pm SEM. Number of animals per group was: C57BL/6J $(n=20,13$, and 6) and DBA/2J $(n=24,15$, and 13) for control, I and 14 days withdrawal groups, respectively.
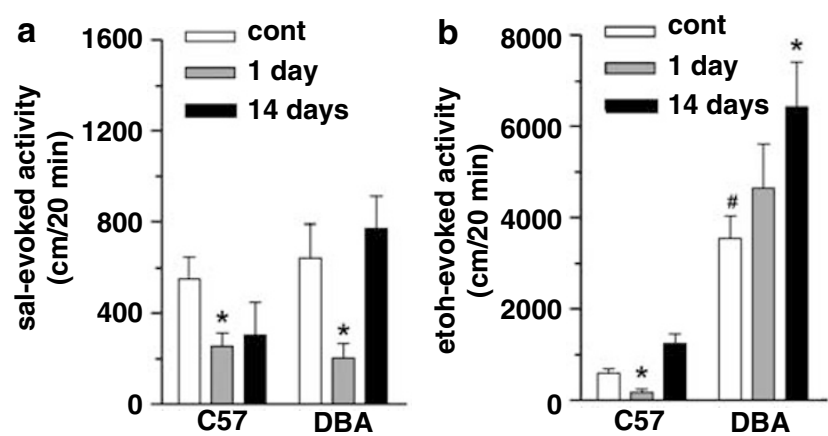

Figure 4 Total ambulatory activity during the $20 \mathrm{~min}$ following saline (a) or $\mathrm{ETOH} 2 \mathrm{~g} / \mathrm{kg}$ i.p. (b) challenge in naïve or $\mathrm{ETOH}$ withdrawn (I or 14 day) C57BL/6J and DBA/2J mice. Values are mean \pm SEM. $* p<0.05$ vs control, ETOH-naïve animals, Mann-Whitney's rank-sum test. Number of animals as in Figure 3.

test). In C57BL/6J mice, withdrawal condition had a significant effect on ETOH-evoked locomotor activity $(\mathrm{H}=16.24$, with $2 \mathrm{df}, p<0.001$, Kruskal-Wallis ANOVA on ranks). Post hoc analysis revealed that ETOH-evoked locomotor activity was decreased in the 1-day withdrawal group $v s$ control mice in this strain. However, at the 14-day withdrawal time point, the locomotor response to ETOH was no longer significantly different from control naïve C57BL/6J mice. A significant effect of withdrawal condition was also detected in $\mathrm{DBA} / 2 \mathrm{~J}$ mice $(\mathrm{H}=6.37$, with $2 \mathrm{df}$, $p=0.041$, Kruskal-Wallis ANOVA on ranks). However, post hoc analysis showed that this effect was due to an enhanced ETOH-evoked response in the long-term withdrawal group compared to the control mice. In contrast to C57BL/6J mice, no significant attenuation of the ETOH-evoked locomotor response was observed at the 1-day withdrawal time point in $\mathrm{DBA} / 2 \mathrm{~J}$ mice.

\section{Microdialysis}

No significant difference in basal or ETOH-evoked DA was observed in control animals that were run at either the 1- or 14-day withdrawal time point. Thus, control animals from both groups were pooled for subsequent statistical analysis.

ETOH $(2.0 \mathrm{~g} / \mathrm{kg})$-evoked DA levels are shown in Figure 5. Basal DA levels did not differ between strains and were unaltered following 1 or 14 days of withdrawal (Figure 6a, no significant main effects of strain, $\mathrm{F}_{(1,82)}=3.361$, $p=0.070$, or withdrawal condition, $\mathrm{F}_{(2,82)}=0.402, p=$ 0.670 , and no significant interaction $\mathrm{F}_{(2,82)}=0.203, p=0.817$, two-factor ANOVA). Although statistical analysis of AUC values revealed a significant effect of strain (Figure 6b, $\left.\mathrm{F}_{(1,82)}=4.126, p=0.045\right)$, post hoc analysis did not reveal a significant strain difference at either withdrawal time. Furthermore, when data were expressed as percentage of baseline, no strain difference was seen. There was, however, a main effect of the withdrawal condition on the ETOHevoked DA response $\left(\mathrm{F}_{(2,82)}=6.037, p=0.004\right)$. Post hoc analysis revealed that this effect was due to the marked inhibition of the ETOH-evoked DA response in the 1-day withdrawal group. No significant strain by withdrawal condition interaction $\left(\mathrm{F}_{(2,82)}=0.483, p=0.619\right)$ was seen.

Since the control animals were not treated with pyrazole, additional experiments were performed in order to test whether pyrazole treatment alters ETOH-evoked accumbens DA when compared to saline. Basal DA levels (nM) were not altered by pyrazole pretreatment (control: $1.46 \pm 0.24, n=6$; pyrazole: $1.70 \pm 0.30, n=6$ ). In addition, the DA response (\% maximum increase from baseline) evoked by a $2 \mathrm{~g} / \mathrm{kg}$ i.p.
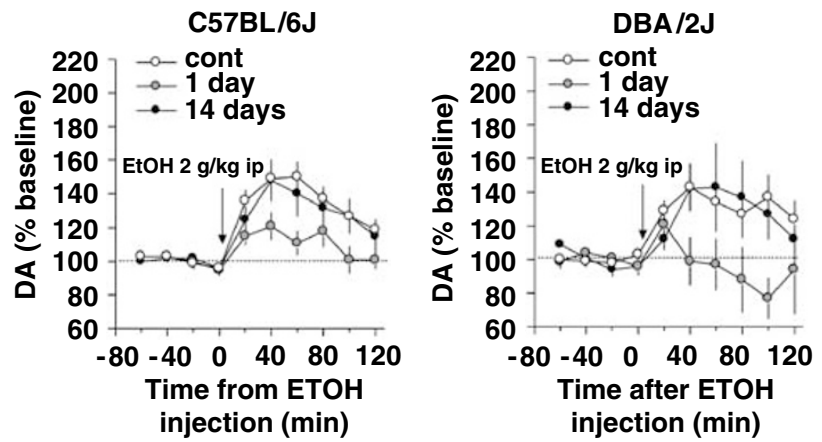

Figure 5 Time course of the effects of an $\mathrm{ETOH} 2 \mathrm{~g} / \mathrm{kg}$ i.p. challenge on dialysate DA levels in the nucleus accumbens of naive or $\mathrm{ETOH}$ withdrawn ( 1 or I 4 day) mice. Values are mean \pm SEM. Number of animals per group was: C57BL/6) $(n=21,17$, and 12) and DBA/2J $(n=20,5$, and 13) for control, I and 14-day withdrawal groups, respectively. 

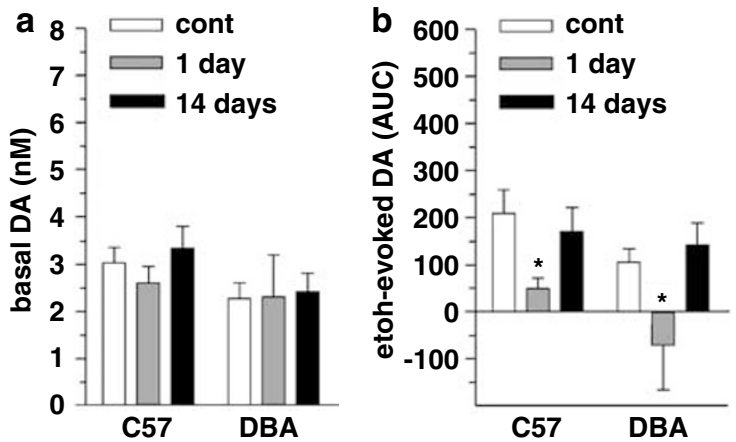

Figure 6 Basal dialysate DA levels (a) and total ETOH-evoked dopamine outflow relative to basal levels (b) after an ETOH $2 \mathrm{~g} / \mathrm{kg}$ i.p. challenge in naïve or $\mathrm{ETOH}$ withdrawn ( I or I 4 day) C57BL/6) and DBA/2] mice. ${ }^{*} p<0.05$ vs control, ETOH -naïve animals, Student-Newman-Keuls test. Number of animals as in Figure 5.

ETOH challenge did not differ between the two treatment groups (control: $114 \pm 3, n=6$; pyrazole: $117 \pm 4, n=6$ ).

\section{DISCUSSION}

The acute administration of ETOH increased locomotor activity in previously naïve DBA/2J and C57BL/6J mice. In agreement with a previous study (Phillips et al, 1994), the magnitude of the behavioral response to ETOH was significantly greater in DBA/2J mice. There was, however, no difference between strains in the magnitude of ETOHevoked DA levels in the nucleus accumbens, indicating that the increased behavioral sensitivity of DBA/2J mice is not associated with an enhanced responsiveness of mesoaccumbal DA neurons to ETOH. Indeed, comparison of the responses to ETOH based on dialysate concentrations of DA in the two strains suggests an inverse relationship between the behavioral and DA response to ETOH. That is, the magnitude of the change in dialysate DA concentration after ETOH, overall, was greater in the C57BL/6J strain which showed a reduced behavioral response to ETOH relative to DBA/2J mice. There have been previous reports of an association between drug-evoked locomotor activity and accumbens DA release in C57BL/6J and DBA/2J mice for amphetamine (Zocchi et al, 1998) and morphine (Murphy et al, 2001). In both studies, C57BL/6J mice showed greater locomotor activation and a greater increase in dialysate DA levels in response to the acute administration of these agents. This association, however, may depend on the drug tested. For example, although several laboratories have shown that DBA/2J mice show enhanced cocaine-evoked locomotion compared to C57BL/6J (Rocha et al, 1998; Phillips et al, 1998), a microdialysis study found no difference in the magnitude of cocaine-evoked DA levels in the nucleus accumbens between these two mouse strains (He and Shippenberg, 2000).

The results obtained on ETOH-evoked DA levels in the nucleus accumbens are supported by a previous in vitro study in which ETOH-stimulated DA cell firing in ventral tegmental area slices of DBA/2J mice was similar to that in C57BL/6J mice (Brodie and Appel, 2000), at least at the same ETOH concentrations used in the present study. Since ventral tegmental DA cells project to the nucleus accumbens, one would predict that increased activity in cell bodies should result in increased terminal release of DA. However, caution should be maintained when comparing in vivo and in vitro models in the two studies. In this regard, however, it is important to note that ventral tegmental DA neurons project not only to the nucleus accumbens but also to other limbic structures including the medial prefrontal cortex and amygdala. Therefore, differences in ETOH-stimulated DA release between the two mouse strains may occur in other brain regions. Finally, it has been suggested that microdialysis is only able to detect changes in tonic (extrasynaptic) DA that is associated with an increase in the number of firing cells as opposed to phasic (synaptic) DA release that is associated with an increase in the firing frequency (Floresco et al, 2003). Therefore, it is possible that the microdialysis technique is limited (Yang et al, 1998) in detecting potential strain differences in the activity of the mesoaccumbal system in response to an ETOH challenge. More detailed studies comparing the firing rate of DA neurons coupled with measurement of extracellular DA concentrations at the terminal regions are necessary to fully address this question.

Locomotor activity in response to saline was decreased in both strains, relative to that of controls, 1 day following the discontinuation of the repeated ETOH intoxication and withdrawal protocol. Spontaneous activity was also reduced at this time point. The depression of activity is most likely attributable to malaise associated with the early phase of ETOH withdrawal. Consistent with this hypothesis, the decrease in spontaneous locomotion was greater in DBA/2J mice, which also exhibited a more severe physical withdrawal syndrome as indicated by the HIC score, than in C57BL/6J mice. At this same withdrawal time point, C57BL/ $6 \mathrm{~J}$ mice exhibited tolerance to the locomotor-activating effects of ETOH. In contrast, DBA/2J exhibited a robust stimulation of locomotor activity in response to the same challenge dose of ETOH. Since spontaneous and salineevoked activity was depressed at this abstinence time point the possibility arises that a nonspecific withdrawal-induced decrease in activity may have contributed to the results obtained. In this regard, however, it is important to note that in contrast to C57BL/6J mice behavioral tolerance was not observed in the DBA/2J strain. Yet, this latter strain not only exhibited a more severe physical withdrawal syndrome than C57BL/6J mice but also exhibited a greater depression of spontaneous activity.

Despite differences in the behavioral response to ETOH, tolerance to the DA-releasing effects of ETOH was apparent in both strains on day one of withdrawal. The observation of a blunted ETOH-evoked DA response at this early withdrawal time point is intriguing. To our knowledge, this is the first description of this effect and the mechanisms involved are unknown. Previous studies have reported no difference in the DA response to an ETOH challenge in dependent (not withdrawn) rats (Rossetti et al, 1993). Another study reported a decrease in basal DA activity during the acute withdrawal phase that was reversed by an acute ETOH challenge (Diana et al, 1993). These findings and those of the present study highlight the plasticity of mesoaccumbal DA neurons and the importance of time-course studies in delineating the neurochemical 
mechanisms mediating the behavioral effects resulting from the repeated administration of ETOH and other drugs of abuse. The fact that both strains differed in their locomotor response to ETOH but exhibited similar decreases in ETOHevoked DA levels in the nucleus accumbens shows that the behavioral and mesoaccumbal DA response to withdrawal can be dissociated. Furthermore, it demonstrates that a dysregulation of presynaptic mesoaccumbal DA neurotransmission cannot account for the alterations in the behavioral effects of ETOH observed during the early phase of withdrawal.

It is important to note that strain differences in blood ETOH levels were observed at the 1-day withdrawal time point. However, these pharmacokinetic differences cannot fully explain the results obtained. C57BL/6J (but not DBA/ 2J) mice had lower blood levels in response to the $2 \mathrm{~g} / \mathrm{kg}$ ETOH challenge compared to ETOH naïve animals. This suggests that ETOH-exposed C57BL/6J mice may metabolize ETOH faster than controls or DBA/2J mice. While this metabolic tolerance is consistent with the observation of behavioral tolerance in C57BL/6J but not in the DBA/2J mice, it cannot explain the observation of tolerance to the ETOH-evoked accumbens DA response in both mouse strains. A lower blood ETOH level after systemic challenge was observed in DBA/2J mice after 14 days withdrawal. The mechanism leading to the more rapid metabolism in this strain awaits clarification. However, it is unlikely that the lower blood levels can explain the lack of a sensitized DA response since this blood level was sufficient for the development of a sensitized behavioral response.

One potential confound in the microdialysis studies is the fact that control animals were not treated with pyrazole. Since pyrazole can inhibit dopamine $\beta$-hydroxylase the possibility arises that the effects attributed to ETOH may, in fact, result from pyrazole-induced alterations in catecholamine synthesis. Three points suggest that this explanation is unlikely. Firstly, following acute pyrazole administration, sustained effects on norepinephrine were only observed in response to a dose of $500 \mathrm{mg} / \mathrm{kg}$, whereas a lower dose were $(100 \mathrm{mg} / \mathrm{kg})$ had only transient effects (MacDonald, 1977). Moreover, repeated daily administration of a lower dose $(50 \mathrm{mg} / \mathrm{kg})$, more in the range of that used in the present study, resulted in changes in norepinephrine only after 6 days of administration and this effect was prevented by concomitant administration of ETOH (Brown et al, 1978). Secondly, in the one study that investigated pyrazoleinduced changes in DA found no changes in DA synthesis or tissue levels in the striatum (MacDonald and Pispa, 1977). Finally, in the present study, microdialysis experiments assessing the effects of pyrazole alone $(68 \mathrm{mg} / \mathrm{kg} /$ day $\times 4$ days) on basal and ETOH-evoked DA levels revealed no differences between pyrazole and control (saline-treated) animals $24 \mathrm{~h}$ following treatment cessation. Given the evidence reviewed above, it is unlikely that the pyrazole treatment alone can explain the lack of ETOHevoked DA response at the $24 \mathrm{~h}$ withdrawal time point. Rather, the effects observed are a direct consequence of the repeated ETOH intoxication schedule.

Most studies investigating the effects of chronic ETOH exposure on the function of the mesolimbic DA system have been conducted during the acute withdrawal phase $(6-8 \mathrm{~h})$, which is characterized by a hyperexcitability and physical withdrawal signs. These studies have consistently shown a profound reduction in the activity of mesolimbic DA neurons that is reversed by resuming ETOH exposure (Rossetti et al, 1992; Diana et al, 1993; Weiss et al, 1996). However, studies examining whether these changes persist beyond the acute withdrawal phase have been lacking. The present data show that regardless of strain, basal DA levels in the nucleus accumbens do not differ from that of control animals 1 day after withdrawal. However, definitive evidence for that conclusion requires the use of quantitative microdialysis techniques, which provide an estimate of extracellular DA concentrations. Nevertheless, the present data suggest that ETOH-induced alterations in basal DA neurotransmission are transient and have normalized by day 1 of withdrawal. The finding, however, of a decreased response of DA neurons to an acute ETOH challenge suggests that there is, in fact, a more persistent dysregulation of mesoaccumbal DA neurotransmission. Regardless of strain, the expression of tolerance was transient. It was observed at day 1 but not at day 14 of withdrawal.

As observed during early abstinence, protracted abstinence was also associated with strain-related differences in the behavioral response to an acute ETOH challenge. At 14 days following the cessation of the repeated intoxication and withdrawal protocol, a marked enhancement of the locomotor-activating effects of ETOH was observed in DBA/ $2 \mathrm{~J}$ mice. In contrast, C57BL/6J strain showed a slight but nonsignificant increase in ETOH-evoked locomotion. It should be noted that the lower number of C57BL/6J in the 14-day withdrawal group may have contributed to the lack of a statistically significant effect. However, this finding is consistent with previous studies showing no (Phillips et al, 1994) or only marginal behavioral sensitization (Lessov et al, 2001) in C57BL/6J mice, in contrast to DBA/2J mice. This strain difference in the ability of repeated ETOH administration to induce behavioral sensitization is consistent with previous studies (Cunningham et al, 1992; Phillips et al, 1994; Lessov et al, 2001). Neither strain exhibited an enhancement of ETOH-evoked DA levels at the 14-day withdrawal time point. Thus, despite the sensitized behavioral response to ETOH in DBA/2J mice, the magnitude of the ETOH-induced increase in DA levels did not differ from that of previously naïve mice. This suggests that failure to see an enhanced DA response to ETOH cannot be attributed to an inability of our repeated ETOH intoxication paradigm to induce behavioral sensitization. An enhanced locomotor response to ETOH was apparent in the DBA/2J strain and an identical ETOH intoxication and withdrawal procedure was previously reported to result in sensitization to other effects of ETOH including withdrawalinduced seizures (Becker et al, 1997). In addition, our studies were conducted in two different mouse strains previously shown to differ dramatically in their behavioral responses to ETOH. Yet, neither strain exhibited an enhancement of ETOH-evoked DA levels.

Studies with other drugs of abuse (eg psychostimulants and opiates) have shown that the expression of an enhanced drug-evoked DA response depends on both the challenge dose employed and the duration of abstinence. Therefore, the failure to observe an enhanced DA response to ETOH may have resulted from the specific experimental conditions employed in the present study. However, additional 
experiments using a lower ETOH challenge dose $(1 \mathrm{~g} / \mathrm{kg})$ as well as a longer withdrawal time (4 weeks) also failed to demonstrate a sensitized DA response to ETOH in C57BL/6J mice (data not shown).

The present findings are of significance in view of two current hypotheses regarding the neurochemical basis of substance abuse. First, it is thought that sensitization of the mesolimbic DA system is a hallmark of all drugs of abuse and that it is critical for the development of the addictive behavior (Robinson and Berridge, 1993). Second, a causal link between the long-term expression of behavioral sensitization and sensitization of the mesolimbic DA system has been suggested (Kalivas and Duffy, 1993a; Vanderschuren and Kalivas, 2000). The failure of repeated ETOH intoxication to induce sensitization of the accumbens DA response suggests that repeated exposure to different drugs of abuse does not necessarily result in a common final neuroadaptation that triggers compulsive drug use or, alternatively, and contrary to a widely held notion, this common final neuroadaptation does not involve sensitization of the mesolimbic DA system (Kalivas et al, 1998; Robinson and Berridge, 2001). Of course, the fact that we failed to see sensitization of the DA response does not prove that it would not occur under other experimental conditions. However, to our knowledge, no study to date has demonstrated a sensitized accumbens DA response after repeated ETOH administration. Interestingly, data showing an increased ETOH-evoked firing of DA neurons in ventral tegmental area slices obtained from C57BL/6J mice after repeated ETOH administration has been reported (Brodie, 2002). This study is intriguing since slices were obtained after $12 \mathrm{~h}$ from the last ETOH administration, when the animals were presumably still in the early phase of withdrawal. It is known that the withdrawal period is a critical factor in the development of sensitization, and that longer withdrawal intervals facilitate sensitization. By contrast, early withdrawal points favor the observation of tolerance phenomena (Kalivas and Duffy, 1993a). Indeed, in our study, a clear tolerance to both the behavioral and neurochemical effects of an ETOH challenge was observed in both mouse strains at the early withdrawal testing point $(24 \mathrm{~h})$. It may be that sensitization in the neuronal cell bodies is not necessarily related to sensitization of neurotransmitter release in the projection areas. In fact, there is some evidence indicating that, at least for psychostimulants, DA sensitization in the perikarya follows a different temporal course than in the terminals. It is observed at early withdrawal stages, is short-lived and does not temporally correlate with behavioral sensitization (Kalivas and Duffy, 1993a, b).

An additional implication of the present results concerns the association of an enhanced accumbens DA response to the long-term expression of behavioral sensitization. It has been hypothesized that the locomotor-activating effects of psychostimulants is related to their ability to stimulate accumbal DA release. Indeed, destruction of DA terminals in this brain region blocks behavioral activation (Kelly and Iversen, 1976). In addition, behavioral sensitization is associated with enhanced accumbens DA release, at least at long withdrawal intervals, since during early withdrawal and especially after a high-dose regimen, tolerance phenomena can mask the observation of the sensitized DA response (Kalivas and Duffy, 1993a). In the case of ETOH, available evidence suggests a role for the DA system in the ability of acute ETOH to stimulate locomotor activity (Phillips and Shen, 1996). Our data suggest that in the case of repeated ETOH intoxication and withdrawal, behavioral stimulation can occur independently of a sensitized accumbens DA response. Indeed, a dissociation between the mechanisms mediating the effects of acute and repeated ETOH on locomotor activity has been previously suggested, based on the observation that DA antagonists block the acute but not the sensitized behavioral response to ETOH (Broadbent et al, 1995). This may suggest that other brain regions or other neurotransmitter systems have a more prominent role in mediating the development and longterm expression of ETOH-induced behavioral sensitization. In this regard, other areas receiving DA projections (eg central amygdala) may be important sites mediating several effects of repeated ETPH administration (McBride, 2002). In addition, repeated ETOH exposure is associated with changes in other neurotransmitter such as glutamate and GABA (Krystal et al, 2003; Roberto et al, 2004; Melendez et al, 2005). The finding that glutamate receptor antagonists block the development and expression of ETOH-evoked behavioral sensitization suggests an important role of this neurotransmitter in the sensitization process (Broadbent and Weitemier, 1999, Broadbent et al, 2003; Meyer and Phillips, 2003).

In summary, we have investigated the ability of repeated ETOH intoxication and withdrawal to induce sensitization of the behavioral and the accumbal DA response to a systemic ETOH challenge. Despite the demonstration of behavioral sensitization, no enhanced DA response to ETOH was observed. The failure of the repeated ETOH treatment to induce DA sensitization was observed in two mouse strains known to differ in their behavioral responses to ETOH. Moreover, clear tolerance to the locomotoractivating effects of ETOH and to ETOH-evoked DA release was observed 1 day after terminating the chronic ETOH treatment regime. These results suggest that, at least for ETOH, the behavioral sensitization associated with protracted withdrawal can be observed in the absence of an enhanced responsiveness of mesoaccumbal DA neurons to an acute ETOH challenge.

\section{ACKNOWLEDGEMENTS}

We thank Vicki Minney for excellent technical support. This study was supported by grants from the National Institute of Alcohol Abuse and Alcoholism, NIH (AA U01 13486-INIA Project) and the National Institute on Drug Abuse Intramural Research Program.

\section{REFERENCES}

Becker HC (1994). Positive relationship between the number of prior ethanol withdrawal episodes and the severity of subsequent withdrawal seizures. Psychopharmacology (Berlin) 116: 26-32.

Becker HC, Diaz-Granados JL, Weathersby RT (1997). Repeated ethanol withdrawal experience increases the severity and duration of subsequent withdrawal seizures in mice. Alcohol 14: $319-326$. 
Broadbent J, Grahame NJ, Cunningham CL (1995). Haloperidol prevents ethanol-stimulated locomotor activity but fails to block sensitization. Psychopharmacology (Berlin) 120: 475-482.

Broadbent J, Kampmueller KM, Koonse SA (2003). Expression of behavioral sensitization to ethanol by DBA/2J mice: the role of NMDA and non-NMDA glutamate receptors. Psychopharmacology (Berin) 167: 225-234.

Broadbent J, Weitemier AZ (1999). Dizocilpine (MK-801) prevents the development of sensitization to ethanol in DBA/2J mice. Alcohol Alcohol 34: 283-288.

Brodie MS (2002). Increased ethanol excitation of dopaminergic neurons of the ventral tegmental area after chronic ethanol treatment. Alcohol Clin Exp Res 26: 1024-1030.

Brodie MS, Appel SB (2000). Dopaminergic neurons in the ventral tegmental area of $\mathrm{C} 57 \mathrm{BL} / 6 \mathrm{~J}$ and $\mathrm{DBA} / 2 \mathrm{~J}$ mice differ in sensitivity to ethanol excitation. Alcohol Clin Exp Res 24: $1120-1124$

Brown FC, Zawad J, Harralson JD (1978). Interactions of pyrazole and ethanol on norepinephrine metabolism in rat brain. J Pharmacol Exp Ther 206: 75-80.

Cadoni C, Di Chiara G (1999). Reciprocal changes in dopamine responsiveness in the nucleus accumbens shell and core and in the dorsal caudate-putamen in rats sensitized to morphine. Neuroscience 90: 447-455.

Cadoni C, Di Chiara G (2000a). Differential changes in accumbens shell and core dopamine in behavioral sensitization to nicotine. Eur J Pharmacol 387: R23-R25.

Cadoni C, Solinas M, Di Chiara G (2000b). Psychostimulant sensitization: differential changes in accumbal shell and core dopamine. Eur J Pharmacol 388: 69-76.

Crabbe JC, Belknap JK (1993). Behavior genetic analyses of drug withdrawal. Alcohol Alcohol Suppl 2: 477-482.

Crabbe JC, Kosobud A, Young ER, Janowsky JS (1983). Polygenic and single-gene determination of responses to ethanol in BXD/ Ty recombinant inbred mouse strains. Neurobehav Toxicol Teratol 5: 181-187.

Cunningham CL, Niehus DR, Malott DH, Prather LK (1992). Genetic differences in the rewarding and activating effects of morphine and ethanol. Psychopharmacology (Berlin) 107: 385-393.

Diana M, Pistis M, Carboni S, Gessa GL, Rossetti ZL (1993). Profound decrement of mesolimbic dopaminergic neuronal activity during ethanol withdrawal syndrome in rats: electrophysiological and biochemical evidence. Proc Natl Acad Sci USA 90: 7966-7969.

Duka T, Gentry J, Malcolm R, Ripley TL, Borlikova G, Stephens DN et al (2004). Consequences of multiple withdrawals from alcohol. Alcohol Clin Exp Res 28: 233-246.

Fish EW, DeBold JF, Miczek KA (2002). Repeated alcohol: behavioral sensitization and alcohol-heightened aggression in mice. Psychopharmacology (Berlin) 160: 39-48.

Floresco SB, West AR, Ash B, Moore H, Grace AA (2003). Afferent modulation of dopamine neuron firing differentially regulates tonic and phasic dopamine transmission. Nat Neurosci 6: 968-973.

He M, Shippenberg TS (2000). Strain differences in basal and cocaine-evoked dopamine dynamics in mouse striatum. J Pharmacol Exp Ther 293: 121-127.

Hoshaw BA, Lewis MJ (2001). Behavioral sensitization to ethanol in rats: evidence from the Sprague-Dawley strain. Pharmacol Biochem Behav 68: 685-690.

Imperato A, Di Chiara G (1986). Preferential stimulation of dopamine release in the nucleus accumbens of freely moving rats by ethanol. J Pharmacol Exp Ther 239: 219-228.

Kalivas PW, Duffy P (1993a). Time course of extracellular dopamine and behavioral sensitization to cocaine. I. Dopamine axon terminals. J Neurosci 13: 266-275.
Kalivas PW, Duffy P (1993b). Time course of extracellular dopamine and behavioral sensitization to cocaine. II. Dopamine perikarya. J Neurosci 13: 276-284.

Kalivas PW, Pierce RC, Cornish J, Sorg BA (1998). A role for sensitization in craving and relapse in cocaine addiction. J Psychopharmacol 12: 49-53.

Kelly PH, Iversen SD (1976). Selective 6OHDA-induced destruction of mesolimbic dopamine neurons: abolition of psychostimulantinduced locomotor activity in rats. Eur J Pharmacol 40: 45-56.

Krystal JH, Petrakis IL, Mason G, Trevisan L, D’Souza DC (2003). $\mathrm{N}$-methyl-D-aspartate glutamate receptors and alcoholism: reward, dependence, treatment, and vulnerability. Pharmacol Ther 99: 79-94.

Lessov CN, Palmer AA, Quick EA, Phillips TJ (2001). Voluntary ethanol drinking in C57BL/6J and DBA/2J mice before and after sensitization to the locomotor stimulant effects of ethanol. Psychopharmacology (Berlin) 155: 91-99.

Liljequist S, Berggren U, Engel J (1981). The effect of catecholamine receptor antagonists on ethanol-induced locomotor stimulation. J Neural Transm 50: 57-67.

MacDonald E (1977). Effects of pyrazole treatment of physical status and brain biogenic amines in rats. Med Biol 55: 101-108.

MacDonald E, Pispa JP (1977). Evidence for inhibition of dopamine-beta-hydroxylase in vivo after sub-acute pyrazole treatment in rats. Med Biol 55: 284-291.

McBride WJ (2002). Central nucleus of the amygdala and the effects of alcohol and alcohol-drinking behavior in rodents. Pharmacol Biochem Behav 71: 509-515.

Melendez RI, Hicks MP, Cagle SS, Kalivas PW (2005). Ethanol exposure decreases glutamate uptake in the nucleus accumbens. Alcohol Clin Exp Res 29: 326-333.

Meyer PJ, Phillips TJ (2003). Bivalent effects of MK-801 on ethanolinduced sensitization do not parallel its effects on ethanolinduced tolerance. Behav Neurosci 117: 641-649.

Murphy NP, Lam HA, Maidment NT (2001). A comparison of morphine-induced locomotor activity and mesolimbic dopamine release in C57BL6, 129Sv and DBA2 mice. J Neurochem 79: 626-635.

Nestby P, Vanderschuren LJ, De Vries TJ, Hogenboom F, Wardeh G, Mulder AH et al (1997). Ethanol, like psychostimulants and morphine, causes long-lasting hyperreactivity of dopamine and acetylcholine neurons of rat nucleus accumbens: possible role in behavioural sensitization. Psychopharmacology (Berlin) 133: 69-76.

Nestby P, Vanderschuren LJ, De Vries TJ, Mulder AH, Wardeh G, Hogenboom F et al (1999). Unrestricted free-choice ethanol selfadministration in rats causes long-term neuroadaptations in the nucleus accumbens and caudate putamen. Psychopharmacology (Berlin) 141: 307-314.

Phillips TJ, Dickinson S, Burkhart-Kasch S (1994). Behavioral sensitization to drug stimulant effects in C57BL/6J and DBA/2J inbred mice. Behav Neurosci 108: 789-803.

Phillips TJ, Huson MG, McKinnon CS (1998). Localization of genes mediating acute and sensitized locomotor responses to cocaine in BXD/Ty recombinant inbred mice. J Neurosci 18: 3023-3034.

Phillips TJ, Shen EH (1996). Neurochemical bases of locomotion and ethanol stimulant effects. Int Rev Neurobiol 39: 243-282.

Pierce RC, Kalivas PW (1997). A circuitry model of the expression of behavioral sensitization to amphetamine-like psychostimulants. Brain Res Brain Res Rev 25: 192-216.

Reid MS, Ho LB, Berger SP (1996). Effects of environmental conditioning on the development of nicotine sensitization: behavioral and neurochemical analysis. Psychopharmacology (Berlin) 126: 301-310.

Risinger FO, Dickinson SD, Cunningham CL (1992). Haloperidol reduces ethanol-induced motor activity stimulation but not conditioned place preference. Psychopharmacology (Berlin) 107: 453-456. 
Roberto M, Schweitzer P, Madamba SG, Stouffer DG, Parsons LH, Siggins GR (2004). Acute and chronic ethanol alter glutamatergic transmission in rat central amygdala: an in vitro and in vivo analysis. J Neurosci 24: 1594-1603.

Robinson TE, Berridge KC (1993). The neural basis of drug craving: an incentive-sensitization theory of addiction. Brain Res Brain Res Rev 18: 247-291.

Robinson TE, Berridge KC (2001). Incentive-sensitization and addiction. Addiction 96: 103-114.

Rocha BA, Odom LA, Barron BA, Ator R, Wild SA, Forster MJ (1998). Differential responsiveness to cocaine in C57BL/6J and DBA/2J mice. Psychopharmacology (Berlin) 138: 82-88.

Rossetti ZL, Hmaidan Y, Diana M, Gessa GL (1993). Lack of tolerance to ethanol-induced dopamine release in the rat ventral striatum. Eur J Pharmacol 231: 203-207.

Rossetti ZL, Melis F, Carboni S, Diana M, Gessa GL (1992). Alcohol withdrawal in rats is associated with a marked fall in extraneuronal dopamine. Alcohol Clin Exp Res 16: 529-532.

Shen EH, Crabbe JC, Phillips TJ (1995). Dopamine antagonist effects on locomotor activity in naive and ethanol-treated FAST and SLOW selected lines of mice. Psychopharmacology (Berlin) 118: $28-36$.

Vanderschuren LJ, Kalivas PW (2000). Alterations in dopaminergic and glutamatergic transmission in the induction and expression of behavioral sensitization: a critical review of preclinical studies. Psychopharmacology (Berlin) 151: 99-120.
Weiss F, Parsons LH, Schulteis G, Hyytia P, Lorang MT, Bloom $\mathrm{FE}$ et al (1996). Ethanol self-administration restores withdrawal-associated deficiencies in accumbal dopamine and 5-hydroxytryptamine release in dependent rats. J Neurosci 16: 3474-3485.

Yang H, Peters JL, Michael AC (1998). Coupled effects of mass transfer and uptake kinetics on in vivo microdialysis of dopamine. J Neurochem 71: 684-692.

Yim HJ, Schallert T, Randall PK, Gonzales RA (1998). Comparison of local and systemic ethanol effects on extracellular dopamine concentration in rat nucleus accumbens by microdialysis. Alcohol Clin Exp Res 22: 367-374.

Yoshimoto K, McBride WJ, Lumeng L, Li TK (1992). Alcohol stimulates the release of dopamine and serotonin in the nucleus accumbens. Alcohol 9: 17-22.

Zapata A, Chefer VI, Ator R, Shippenberg TS, Rocha BA (2003). Behavioural sensitization and enhanced dopamine response in the nucleus accumbens after intravenous cocaine self-administration in mice. Eur J Neurosci 17: 590-596.

Zapata A, Witkin JM, Shippenberg TS (2001). Selective D3 receptor agonist effects of (+)-PD 128907 on dialysate dopamine at low doses. Neuropharmacology 41: 351-359.

Zocchi A, Orsini C, Cabib S, Puglisi-Allegra S (1998). Parallel strain-dependent effect of amphetamine on locomotor activity and dopamine release in the nucleus accumbens: an in vivo study in mice. Neuroscience 82: 521-528. 